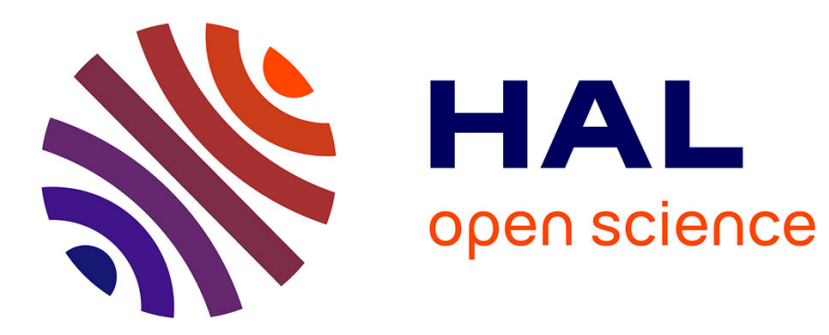

\title{
Online prediction of needle shape deformation in moving soft tissues from visual feedback
}

\author{
Jason Chevrie, Alexandre Krupa, Marie Babel
}

\section{To cite this version:}

Jason Chevrie, Alexandre Krupa, Marie Babel. Online prediction of needle shape deformation in moving soft tissues from visual feedback. IEEE/RSJ Int. Conf. on Intelligent Robots and Systems, IROS'16, Oct 2016, Daejeon, South Korea. pp.2357-2362. hal-01355484

\author{
HAL Id: hal-01355484 \\ https://hal.inria.fr/hal-01355484
}

Submitted on 23 Aug 2016

HAL is a multi-disciplinary open access archive for the deposit and dissemination of scientific research documents, whether they are published or not. The documents may come from teaching and research institutions in France or abroad, or from public or private research centers.
L'archive ouverte pluridisciplinaire HAL, est destinée au dépôt et à la diffusion de documents scientifiques de niveau recherche, publiés ou non, émanant des établissements d'enseignement et de recherche français ou étrangers, des laboratoires publics ou privés. 


\title{
Online prediction of needle shape deformation in moving soft tissues from visual feedback
}

\author{
Jason Chevrie ${ }^{1}$, Alexandre Krupa ${ }^{2}$, Marie Babel $^{3}$
}

\begin{abstract}
With the increasing number of clinical interventions using needle shaped tools, robotic control of needle insertion procedures has been an active research field for many years. In this work we propose a 3D model of a flexible needle that takes into account tissue deformations in order to predict the needle shape and trajectory when it is inserted using a robotic arm. To account for tissue displacements, we designed a method based on visual feedback that updates the interaction model between the needle and the tissue using an unscented Kalman filter. Results obtained from several needle insertions in a soft tissue phantom showed that the method gives good performance in terms of needle trajectory prediction. This model was also considered in a closed-loop control approach to allow automatic reaching of a target.
\end{abstract}

\section{INTRODUCTION}

Image-guided surgical procedures using needle shaped tools have become common minimally invasive interventions for diagnosis or treatment of cancerous tissue [1]. Accurate placement of the tip of the tool in soft tissues is very important to avoid misdiagnosis or destruction of healthy tissues. With current imaging modalities a trade-off has to be made between image acquisition rate and quality. Ultrasound (US) modality offers fast acquisition rates but at the expense of a low image quality. On the contrary MRI or CT-scans offer good quality 3D images of the needle and tissues but the required acquisition time does not allow real-time tracking without degradation of the image quality. In both cases the modeling of the interaction between the tool and the tissue is of great importance. In the first case (US) it can be used to facilitate the needle tracking by providing an initial guess of the needle position in the image, thus reducing the size of the search area, the tracking computation time and the risks of aberrant detection. While in the second case (MRI and CT) it can provide a prediction of the needle position between two image acquisitions. These two aspects are crucial points toward a safe image-guided automatic control of needle manipulators.

Flexible needle insertion modeling has thus been an active research field [2], ranging from simple kinematic models [3] to complex finite element modeling [4]. The first kinematic model, that approximated only the tip motion of a beveledtip needle by a unicycle or bicycle model [3], considered the simple case of very flexible wires embedded in static hard tissues. Many parameters have then been added to

\footnotetext{
${ }^{1}$ Jason Chevrie is with Université de Rennes 1 and IRISA, France, jason.chevrie@irisa.fr

${ }^{2}$ Alexandre Krupa is with Inria Rennes - Bretagne Atlantique and IRISA, France, alexandre.krupaeinria.fr

${ }^{3}$ Marie Babel is with Insa Rennes and IRISA, France, marie.babeleirisa.fr
}

this model to reduce its prediction error [5] and cope with softer tissue [6]. An energetic approach using the RayleighRitz method was also used by Misra et al. [7] to take into account the interaction between the needle and the tissues all along the needle shaft and at the bevel. However, physiological motions of the patient, such as breathing, can induce needle displacement and deformation all along the shaft [8]. This should be even more true with the very flexible beveled needles that are required for this kind of approaches, typically thin nitinol wires, since traditional needles exhibit too low natural curvature in real tissues [9]. Most of the state-of-the-art work focused on tip-based needle steering only considers the case of still tissues or, at most, motion of virtual targets and obstacles. Moreira et al. [10] only recently pointed out the fact that the feasibility of tip-based needle steering in moving tissues still has to be assessed. They showed that tip-based control could be performed under axial tissue motion, i.e. in the same direction as the insertion. Lateral motion, however, were not considered.

In this work we first propose a model that can fully model the 3D behavior of the needle and tissues. It provides the possibility to move both needle and tissues in 3D space and model the resulting shape of the needle. The model is compared to real needle insertions performed with different needles in gelatin phantom. Then we also propose and evaluate a method to estimate the displacement of the tissues using visual feedback. This method was used with different update rates to test the compatibility with rather slow medical imaging modalities like 3D ultrasound in the case of motorized US probes.

The paper is organized as follows: Section II presents the $3 \mathrm{D}$ model that we propose to model the needle and tissue deformation around the needle path. It also details the algorithm that we designed to update the model from visual feedback. We present in Section III the experiments that we conducted to assess the performance of the model and the method used to perform the online update of the model parameters. The algorithm was then used in a targeting task under lateral tissue motion. Finally, conclusions and future work are presented and discussed in section IV.

\section{METHOD}

\section{A. Needle Modeling}

In this section we present a model of the needle and tissue based on the Rayleigh-Ritz method. This model takes into account the interaction of the needle and tissues along the shaft of the needle and the geometry of the needle tip. It can be used to model both stiff and flexible needles and symmetric or asymmetric bevel geometry. The model is made 


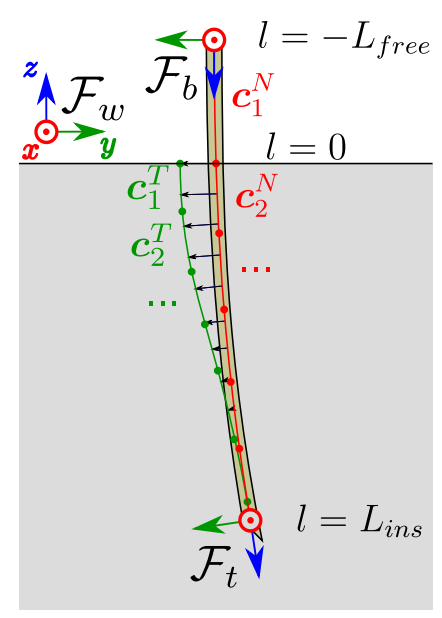

Fig. 1. Needle modeling: needle is red, rest position of the path cut in the tissue is green and tissue surface is black.

up of two parts, one for the needle and one for the tissue. A representation of the model is drawn on Fig. 1.

Let $l$ be the curvilinear coordinate parameter along the needle. We take the convention that $l=0$ at the insertion point, such that $l>0$ corresponds to the part of the needle that is in the tissue and $l<0$ corresponds to the needle outside the tissue. We denote $L_{\text {free }}$ the length outside the tissue and $L_{i n s}$ the length inside the tissue. The needle of length $L$ is modeled as a one dimensional beam represented by a spline curve $c^{N}$ of order $r$ containing $n$ segments:

$$
\begin{aligned}
\boldsymbol{c}^{N}(l) & =\sum_{i=1}^{n} \boldsymbol{c}_{i}^{N}(l), \\
\boldsymbol{c}_{i}^{N}(l) & =\chi_{\boldsymbol{c}_{i}^{N}}(l) M_{i}\left[\begin{array}{lllll}
1 & l & \ldots & l^{r}
\end{array}\right]^{T},
\end{aligned}
$$

where $c^{N}(l) \in \mathbb{R}^{3}$ is the position of a point of the needle at the curvilinear coordinate $l, M_{i} \in \mathbb{R}^{3 \times(r+1)}$ is a matrix containing the coefficients of the polynomial curve $c_{i}^{N}$ and $\chi_{\boldsymbol{c}_{i}^{N}}$ is the characteristic function of the curve, i.e. it takes the value 1 on the definition domain of the curve and 0 elsewhere. Note that the parameters $n$ and $r$ can be chosen to adapt the modeling accuracy and computational complexity.

According to the Euler-Bernouilli beam model, the bending energy $E_{B}$ of the needle can be expressed as

$$
E_{B}=\frac{E I}{2} \int_{-L_{\text {free }}}^{L_{\text {ins }}}\left(\frac{\mathrm{d}^{2} \boldsymbol{c}^{N}(l)}{\mathrm{d} l^{2}}\right)^{2} d l,
$$

where $E$ is the Young's modulus of the needle and $I$ its second moment of area.

We model the tissue by the rest position of the path that the needle cut during the insertion, i.e. the shape of the resulting cut path when the needle is removed from the tissue and does not exert any force on the tissue anymore. This path is also modeled as a spline curve $\boldsymbol{c}^{T}(l)$ (see the green path on Fig. 1). Since the position of the needle corresponds to the current deformed position of the cut path, the tissues exert a resulting force at each point of the needle where it gets away from the rest position of the cut path. For simplicity we assume that the tissues have an elastic behavior, i.e. the exerted force is proportional to the displacement of the tissue. This should be a good approximation as long as the needle remains near the rest cut path, what should be ensured in practice to avoid tissue damage. The resulting force exerted on a segment of the needle between curvilinear coordinates $l_{1}$ and $l_{2}$ will thus be expressed as

$$
\boldsymbol{F}\left(l_{1}, l_{2}\right)=-K_{T} \int_{l_{1}}^{l_{2}} \boldsymbol{c}^{N}(l)-\boldsymbol{c}^{T}(l) d l
$$

where $K_{T}$ denotes the interaction stiffness per unit length.

The energy that is stored in the tissue due to the needle displacement can thus be expressed as

$$
E_{T}=\frac{K_{T}}{2} \int_{0}^{L_{i n s}}\left\|\boldsymbol{c}^{N}(l)-\boldsymbol{c}^{T}(l)\right\|^{2} d l
$$

It has been shown in [11] that the bending energy and tissue deformation energy are sufficient to represent the quasi-totality of the energy stored in the system. So we compute the shape of the needle using the Rayleigh-Ritz method and only considering these two terms. Continuity constraints up to order two between the needle segments are added. We also add the constraints imposed by the needle holder, which fix the needle base position $\boldsymbol{p}_{b}$ and direction $\boldsymbol{d}_{b}$ :

$$
\begin{aligned}
\boldsymbol{c}^{N}\left(-L_{\text {free }}\right) & =\boldsymbol{p}_{b}, \\
\frac{\mathrm{d} \boldsymbol{c}^{N}}{\mathrm{~d} l}\left(-L_{\text {free }}\right) & =\boldsymbol{d}_{b} .
\end{aligned}
$$

The system is then solved as a minimization problem under constraints.

$$
\left\{\begin{array}{c}
\min _{\boldsymbol{m}} E_{B}+E_{T}, \\
\boldsymbol{A} \boldsymbol{m}=\boldsymbol{b}
\end{array}\right.
$$

where $\boldsymbol{m}$ is a vector containing all the coefficients of the matrices $\boldsymbol{M}_{i}$. Matrix $\boldsymbol{A}$ and vector $\boldsymbol{b}$ contain the constraints (6), (7) and the continuity constraints.

As the needle advances in the tissue, the cut path is updated by adding new segments to the spline. To take into account the specific geometry of the needle tip, we choose the new segment such that it links the end of the previous segment to the location of the very tip of the needle, i.e. where the cut occurs in the tissue. In the case of a symmetric tip, the cut path is aligned with the needle axis. In the case of a beveled tip, it is shifted with respect to the needle axis, leading to the creation of a force that pull the needle toward the bevel direction.

Experiments have shown that inserting the needle is sufficient to break the stiction and reset the lag between the tip rotation and the base rotation along the needle shaft caused by torsional friction [12]. Hence we choose here to assume that the tip follows the rotation of the base without lag.

\section{B. Model Update}

We use an unscented Kalman filter (UKF) [13][14] to update the lateral position of the tissue. The UKF provides a higher order of approximation for non-linear systems than the extended Kalman filter while the computation is similar with both methods when dealing with numerical systems [14]. 
We consider the filter states $\mathbf{x} \in \mathbb{R}^{2}$ corresponding to the two lateral translations of the tissue in directions $x$ and $y$ of the world frame $\mathcal{F}_{w}$ (see Fig. 1). In our model these translations are applied to the whole spline defining the rest cut path. We note $\boldsymbol{P}_{x}$ the state covariance matrix. The measures are $\mathbf{y}=\left[\begin{array}{lll}\boldsymbol{p}_{1}{ }^{T} & \ldots & \boldsymbol{p}_{N}{ }^{T}\end{array}\right]^{T}$, where the $\boldsymbol{p}_{i}$ are $N$ points on the inserted part of the needle. We note $l_{i}$ the curvilinear coordinate of point $\boldsymbol{p}_{i}$ on the needle. These points are provided by a visual tracking of the needle. The state representation of the UKF is then given by

$$
\begin{aligned}
\mathbf{x}(k+1) & =\mathbf{x}(k)+\boldsymbol{w}(k), \\
\mathbf{y}(k) & =h(\mathbf{x}(k))+\boldsymbol{n}(k),
\end{aligned}
$$

where $\boldsymbol{w}$ is the process noise, $\boldsymbol{n}$ is the measure noise and $h$ is the relationship between the tissue motion and measured needle points. One advantage of the UKF is that it does not require to know an analytic formulation for $h$, as long as our model provides a numerical way to compute the measures from the states. In our case, our model of the needle allows computing the estimated position of the measured points $\hat{\mathbf{y}}$ via (8) directly from the position of the rest cut path (state) and the pose of the needle base (given by robot odometry).

The position of the rest cut path is then updated according to the new estimate $\hat{\mathbf{x}}(k+1)$ provided by the well-known Kalman filter equations

$$
\begin{aligned}
\hat{\mathbf{x}}(k+1) & =\hat{\mathbf{x}}(k)+\boldsymbol{K}(\mathbf{y}(k+1)-\hat{\mathbf{y}}(k+1)), \\
\boldsymbol{P}_{x}(k+1) & =\boldsymbol{P}_{x}^{-}(k)-\boldsymbol{K} \boldsymbol{P}_{\tilde{y} \tilde{y}} \boldsymbol{K}^{T}, \\
\boldsymbol{K} & =\boldsymbol{P}_{x y} \boldsymbol{P}_{\tilde{y} \tilde{y}}^{-1},
\end{aligned}
$$

where $\boldsymbol{P}_{x}^{-}(k)$ is the predicted state covariance matrix, $\boldsymbol{P}_{x y}$ is the covariance matrix between the states and the measures and $\boldsymbol{P}_{\tilde{y} \tilde{y}}$ is the covariance matrix of the innovation.

The details of the computation of $\boldsymbol{P}_{x}^{-}(k), \boldsymbol{P}_{x y}$ and $\boldsymbol{P}_{\tilde{y} \tilde{y}}$ in the UKF can be found in the literature [13] [14].

Note that this method allows a high flexibility regarding the measurements and the tracking algorithm. Indeed, it is independent of the imaging modality, provided that a measure of the needle position can be acquired. Moreover, the number of tracked points and their position along the needle can vary through time. This allows to perform an update even when the needle is only partially visible in the images, like can for example be the case in ultrasound images when shadows appears due to bones or a lack of gel.

\section{EXPERIMENTAL RESULTS}

This section presents the experiments that we performed to validate our needle insertion model and our update method. We used a similar setup as in [15], where a six degrees of freedom manipulator is used to hold the needle and two orthogonal calibrated cameras are used to provide a visual feedback of the inserted needle. We also present and discuss the results in this section.

\section{A. Model validation}

To validate our model, we made the comparison between the prediction of our needle model and real insertions. We tested three different needles inserted in a home-made gelatin phantom. The characteristics of the different needles are shown in table I. The effective length of the needle that remains outside the needle holder was measured and the other characteristics were those provided by the manufacturer. We measured the stiffness of the phantoms using elastography [16] and found a Young's modulus of $45 \mathrm{kPa}$.

We performed insertions of $10 \mathrm{~cm}$ in three scenarios:

- Scenario 1: the needle is inserted along the $z$ direction of the base frame $\mathcal{F}_{b}$ (see Fig. 1), corresponding to its shaft direction.

- Scenario 2: the needle is only inserted $2 \mathrm{~cm}$ along its shaft direction. The needle base is then moved $2 \mathrm{~mm}$ in the $y$ lateral direction before starting again the insertion in the $z$ direction.

- Scenario 3: like scenario 2 except that the needle base is translated in the opposite lateral direction.

Five insertions were performed for each combination of scenario and needle, while avoiding to cross previous insertions. Fig. 2 shows for each case the mean measured deflection of the tip, i.e. the orthogonal distance between the tip and the initial axis of the needle. As expected we can see that the lateral translation of the needle base during the insertion has a great influence on the final deflection .

The model was then compared to the mean trajectory obtained from these five insertions. For the needle modeling we chose to use polynomials of order 3 and divided the needle in segments of $1 \mathrm{~cm}$. For the tissue modeling we used $1 \mathrm{~mm}$ long polynomials of order 1 (straight lines). In each case the parameter $K_{T}$ was optimized to give the best fit between the prediction and the real deflection. The deflection of the needle trajectory obtained from the model is shown on Fig.2. We can see that the model gives a good fit to the mean insertion trajectories. We found a mean value for the optimal value of $K_{T}=3203 \pm 1614 \mathrm{~N} / \mathrm{m}^{2}$. The high standard deviation in the optimal values of $K_{T}$ can certainly be explained by the fact that the insertions where performed at different locations in the phantom, such that $K_{T}$ should actually be different for each of these locations.

\section{B. Tissue motion tracking}

To assess the quality of our model and update method we performed insertions of the biopsy needle while moving the phantom. We compared the prediction obtained with different model update methods:

- Case 1: the needle is modeled as a straight rigid needle.

- Case 2: the needle is modeled using our model of flexible needle. The cut path extremity is updated without visual measure to correspond to the very tip of the needle model (as explained in section II-A).

- Case 3: similar to case 2, except that the cut path extremity is updated from visual feedback to correspond to the measured position of the tip of the needle.

- Case 4: similar to case 2 with the additional update of the tissue motion using the UKF.

- Case 5: similar to case 3 with the additional update of the tissue motion using the UKF.

For each case the pose of the base of the needle model is updated using the odometry of the robot. 
TABLE I

CHARACTERISTICS OF THE NEEDLES USED IN THE EXPERIMENTS

\begin{tabular}{|c|c|c|c|c|c|c|c|}
\hline Needle type & Reference & Young's modulus & Outer diameter & Inner diameter & Length $(\mathrm{cm})$ & TipType & Tip angle \\
\hline Chiba biopsy needle & Angiotech MCN2208 & $200 \mathrm{GPa}$ & $22 \mathrm{G}(0.7 \mathrm{~mm})$ & $0.48 \mathrm{~mm}$ & 12.6 & Chiba & $25^{\circ}$ \\
\hline Chiba biopsy stylet & Angiotech MCN2208 & $200 \mathrm{GPa}$ & $26 \mathrm{sG}(0.48 \mathrm{~mm})$ & 0.0 & 14.6 & Chiba & $25^{\circ}$ \\
\hline Greene biopsy stylet & Angiotech ISN1915 & $200 \mathrm{GPa}$ & $19 \mathrm{G}(0.97 \mathrm{~mm})$ & 0.0 & 10.8 & Trocar tip & $15^{\circ}$ \\
\hline
\end{tabular}

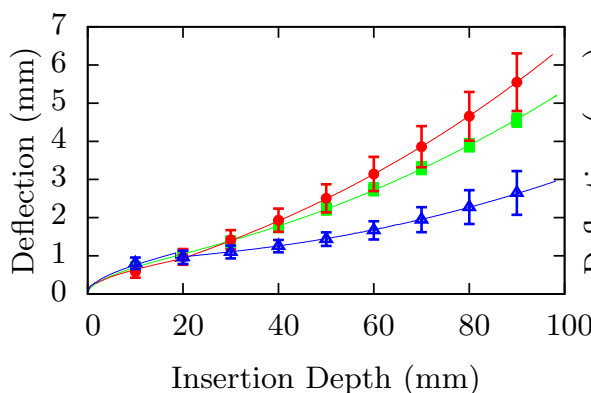

(a) Biopsy needle

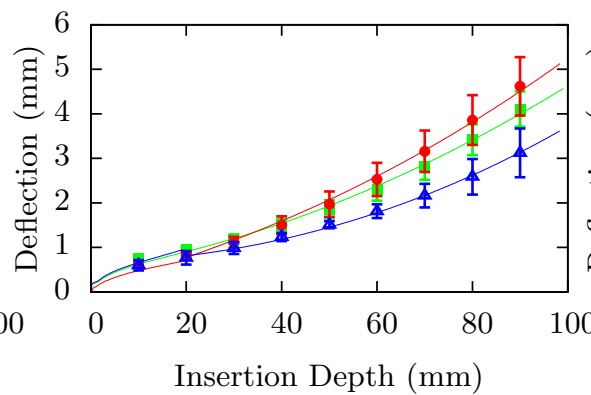

(b) Biopsy stylet

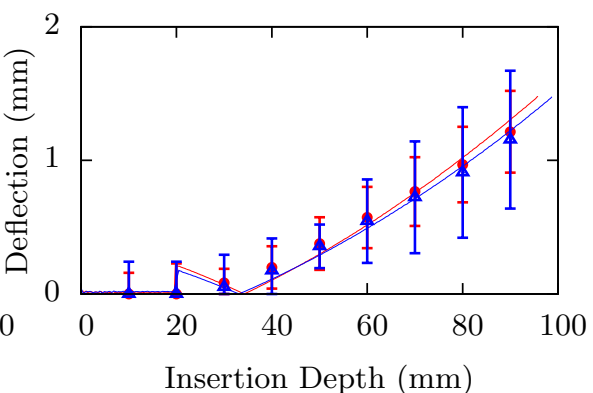

(c) Symmetric needle

Fig. 2. Comparison between model and real needle deflection. Mean experimental values are acquired every centimeter and model deviation is represented with lines: scenario 1 is green, scenario 2 is red and scenario 3 is blue.

We initialized the UKF process noise variance with $\sigma_{w}^{2}=1 \times 10^{-8} \mathrm{~m}^{2}$. As the cameras used for the needle tracking are orthogonal to each other, the accuracy of the stereo vision system had the same value of around $0.25 \mathrm{~mm}$ in each direction. So we set the noise covariance matrix of the UKF as a diagonal matrix with diagonal elements $\sigma_{n}^{2}=\left(2.5 \times 10^{-4}\right)^{2} \mathrm{~m}^{2}$. The stiffness per unit length of the model was set to the mean value found previously, i.e. $K_{T}=3202 \mathrm{~N} / \mathrm{m}^{2}$. For each frame the measured needle points are chosen such that they are spaced by $5 \mathrm{~mm}$ from each other and include the tip point.

We performed three insertions of the needle along its shaft direction, corresponding to scenario 1 of previous section, with an insertion velocity of $5 \mathrm{~mm} / \mathrm{s}$, while applying manual lateral translations to the phantom. The ground truth data of the phantom motion were acquired by tracking four black dots pasted on each visible side of the container (see Fig.4).

Fig. 4 shows a sampled sequence of the camera views during the first experiments with the model rest cut paths for each case (except case 1 that doesn't have tissue modeling). We can see that in case 2 (green) the cut path follows a curve, corresponding to the classical behavior of a flexible beveled needle inserted in still tissues. However this path stays fixed in the world frame and does not follow any of the displacement of the tissue. In case 3 (blue) the shape of the cut path follows the trajectory of the needle tip in space but the path that is already defined does not follow the motion of the phantom. This leads to a final estimated cut path that does not correspond to the reality. The needle shape that is computed from this cut path tends to have an unwanted deformation toward the previous position of the tissues while its tip tends to remain near the real position of the tip. For cases 4 and 5 (red and yellow respectively) the path follows the tissue displacements during the motion. We can see that the final paths stay near the observed needle but are slightly shifted on its side, meaning that the tissues are applying a force on the needle.

The translations that we applied on the tissues by manually moving the phantom during the first experiment are shown on Fig. 3a and the instantaneous positioning error between the modeled tip and the measured tip is shown on Fig. 3b. We can see that the errors for the non-updated models (cases 1 and 2) tend to correspond to the motion of the tissues, while the errors remain low at all time for the models that are updated with the UKF (cases 4 and 5).

To evaluate the possibility to use our model and update algorithm as a prediction tool, we compared the quality of the prediction provided in each case. We considered the case of a prediction after an insertion step of $1 \mathrm{~cm}$. At each timestep we compared the future measured position of the tip to the prediction obtained with the model without update from visual feedback during the $1 \mathrm{~cm}$ prediction step. The prediction error is shown on Fig. 3c. We can see that when using the UKF the prediction error stays low if the tissues are almost not moving and becomes larger when tissue motion occurred during the prediction step.

To see if our method can be used with a slower imaging modality, like 3D ultrasound imaging or fast MRI slice acquisition, we emulated a $1 \mathrm{~Hz}$ acquisition rate system, corresponding to typical values of volume acquisition time with a motorized 3D ultrasound probe. Our acquisition system has an acquisition rate of 30 frames per second so we run the tissue motion update by taking only one frame every 30 frames. The process noise variance was here increased to $\sigma_{w}^{2}=3 \times 10^{-6} \mathrm{~m}^{2}$ to take into account the greater variability in the motion.

We computed the same instantaneous tip position error and the $1 \mathrm{~cm}$ prediction error as defined previously. The results are shown in Fig. $3 \mathrm{~d}$ and Fig. 3e respectively. We can see that both errors tend to increase when important tissue motion occurs between two acquisitions, which is the case for example between $8 \mathrm{~s}$ and $13 \mathrm{~s}$. However this error is greatly reduced by the UKF at each new acquisition. In the case where the tissue motion is slow, we can see that the error is similar to what is obtained with a high image framerate. 

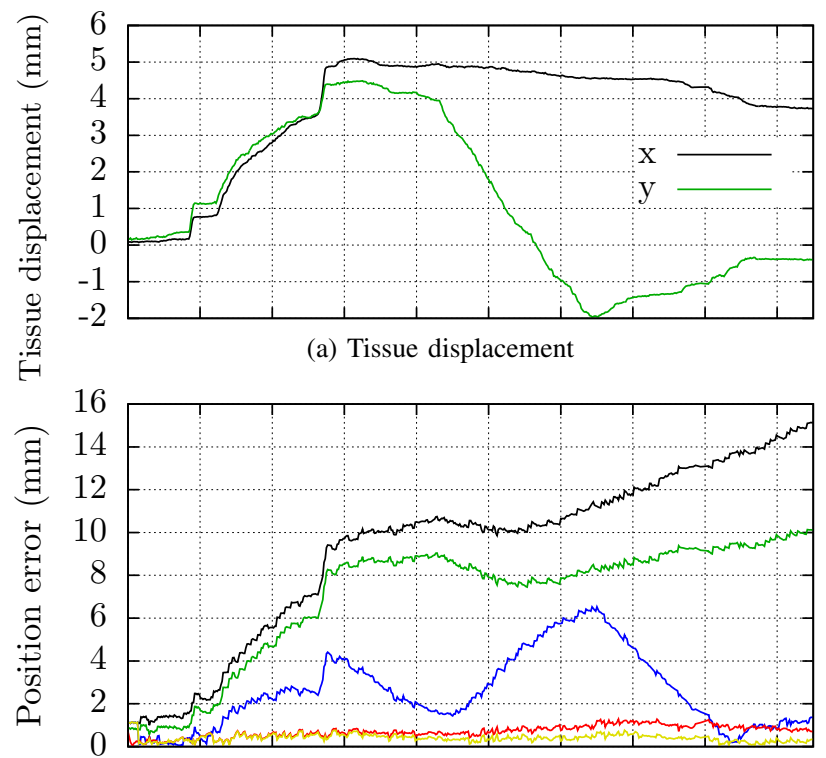

(b) Instantaneous position error between measured and estimated tip position.

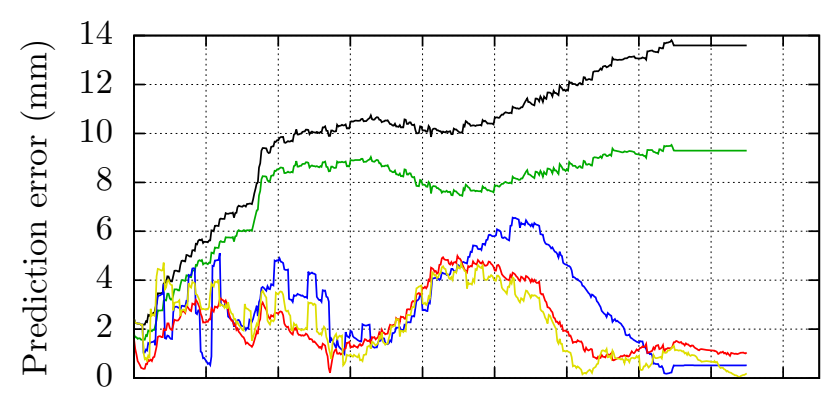

(c) Prediction error between measured tip position and predicted tip position $1 \mathrm{~cm}$ deeper from current position

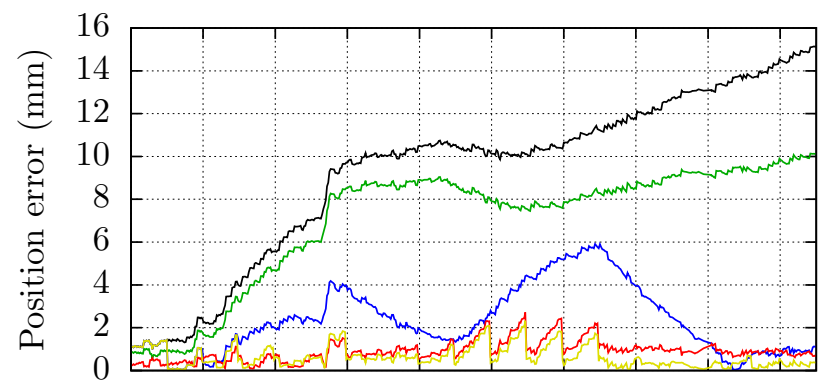

(d) Instantaneous position error between measured and estimated tip position with acquisition rate of $1 \mathrm{~Hz}$.

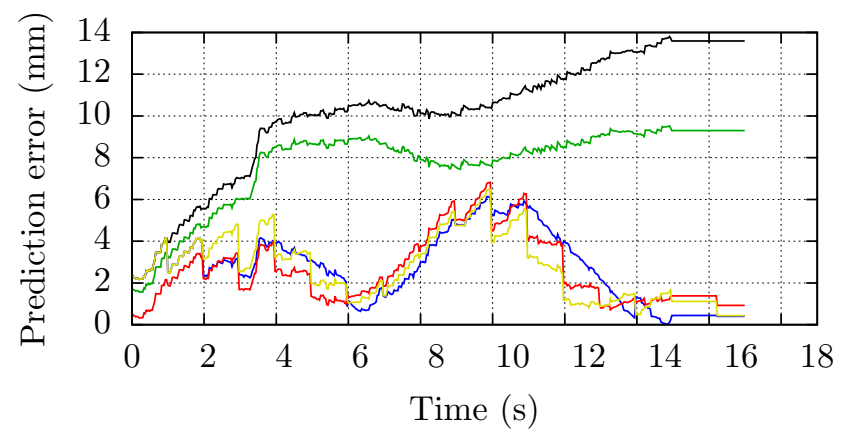

(e) Prediction error between measured tip position and predicted tip position $1 \mathrm{~cm}$ deeper from current position with acquisition rate of $1 \mathrm{~Hz}$.

Fig. 3. Norm of the tip position error for the first experiment. Case 1: black, Case 2: green, Case 3: blue, Case 4: red, Case 5: yellow

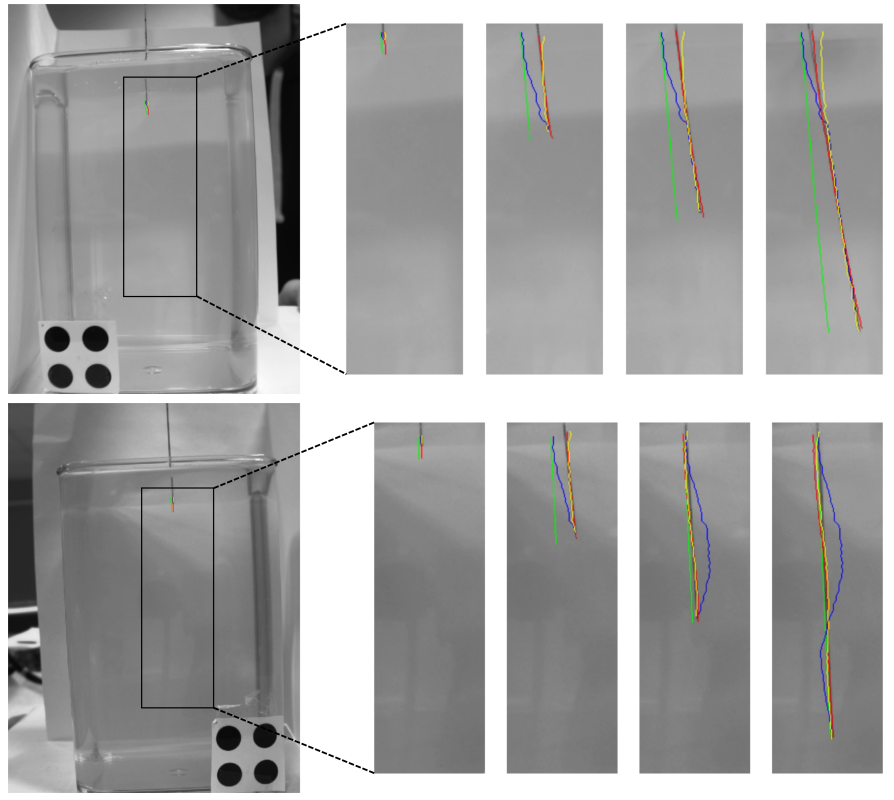

Fig. 4. Sequence of estimated cut paths. Front camera view is on top and side camera view is on the bottom. Timing from left to right: $0.5 \mathrm{~s}, 6 \mathrm{~s}, 13 \mathrm{~s}$, 18s. Case 2: green, Case 3: blue, Case 4: red, Case 5: yellow

\section{TABLE II}

MEAN INSTANTANEOUS POSITION ERRORS AND PREDICTION ERRORS AT $1 \mathrm{~cm}$ FOR THE DIFFERENT UPDATE METHODS DEFINED IN SECTION III-B

\begin{tabular}{c|c|c|c|c|} 
& \multicolumn{2}{|c|}{ Position error $(\mathrm{mm})$} & \multicolumn{2}{c|}{ Prediction error $(\mathrm{mm})$} \\
Framerate & $30 \mathrm{~Hz}$ & $1 \mathrm{~Hz}$ & $30 \mathrm{~Hz}$ & $1 \mathrm{~Hz}$ \\
\hline Case 1 & $5.9 \pm 3.9$ & $5.9 \pm 3.9$ & $6.0 \pm 3.5$ & $6.0 \pm 3.5$ \\
Case 2 & $6.1 \pm 3.0$ & $6.1 \pm 3.0$ & $6.2 \pm 2.5$ & $6.2 \pm 2.5$ \\
Case 3 & $2.1 \pm 1.6$ & $1.9 \pm 1.5$ & $2.5 \pm 1.7$ & $2.4 \pm 1.7$ \\
Case 4 & $0.6 \pm 0.3$ & $0.9 \pm 0.5$ & $2.0 \pm 1.4$ & $2.5 \pm 1.8$ \\
Case 5 & $0.4 \pm 0.2$ & $0.7 \pm 0.5$ & $1.9 \pm 1.4$ & $2.3 \pm 1.7$ \\
\hline
\end{tabular}

Table II recaps the average positioning errors and prediction errors obtained with the different methods during the experiments. As seen previously we can observe that the more the model is updated from visual measures the more the errors are reduced.

\section{Targeting}

In this section we present experiments that we performed to test our model and estimators in a targeting task with the presence of tissue motion.

We used the same control law as presented in [15], which we briefly recall here. The kinematic motion of the needle base, described by its velocity screw vector $\boldsymbol{V}_{b}$, is controlled to obtain a desired velocity of the needle tip $\boldsymbol{v}_{t}$ using:

$$
\boldsymbol{V}_{b}={ }^{t} \boldsymbol{J}_{b}^{+} \boldsymbol{v}_{t},
$$

where ${ }^{t} \boldsymbol{J}_{b}^{+}$denotes the pseudo-inverse of the Jacobian between the tip velocity and the base velocity screw vector. This Jacobian is numerically obtained in real-time from the current updated state of the needle model. The desired tip velocity is computed from visual feedback such that it always points toward the target and has a norm of $2 \mathrm{~mm} / \mathrm{s}$. Additionally the rotation of the needle along its shaft is controlled such that the bevel is always in the direction of the 


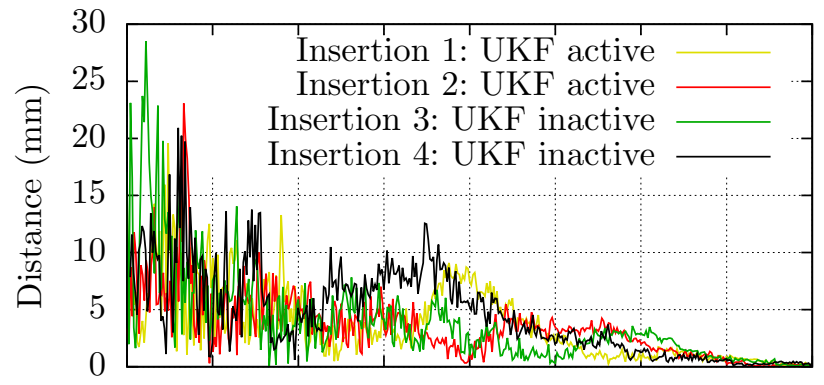

(a) Measure using the visual tracking

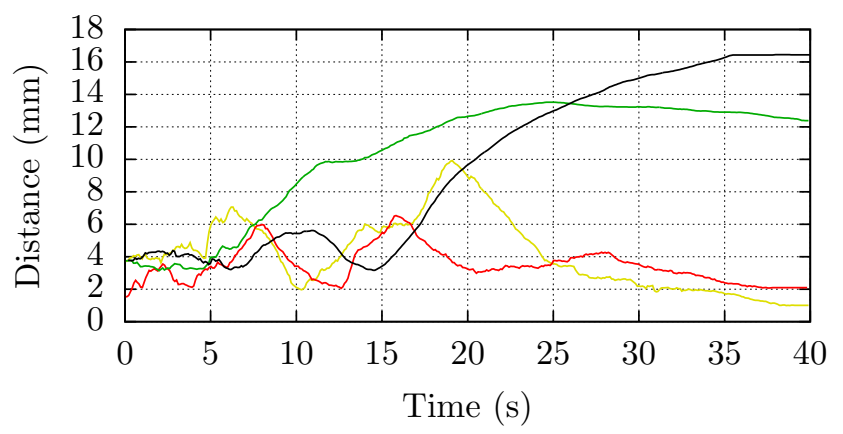

(b) Measure using the model

Fig. 5. Orthogonal distance between the needle tip axis and target

target. The controller is stopped once the needle tip reaches the target level.

A virtual target is defined before each insertion at a fixed location in space such that it is $8 \mathrm{~cm}$ under the tissue surface and $4 \mathrm{~mm}$ away from the initial needle axis. This way a motion of the phantom displaces the needle and leads to a motion of the target with respect to the needle. We performed two insertions with the update using the UKF and two insertions without using the UKF. The phantom was moved manually during the first half of the insertion.

Fig. 5 shows the orthogonal distance between the needle tip axis and the target, both from measures and model. Note that the measures are noisy at the beginning of the insertion because small noisy variations in the measured tip orientation lead to large motion of the needle axis near the distant target. We can see that the target is reached in each case. However the model is far from the target when the update was not active. The targeting task can still perform well in that situation due to the robustness of the control law with respect to modeling errors in the Jacobian ${ }^{t} \boldsymbol{J}_{b}$. Nevertheless the updated model is the only one that fits the observations and can allow to perform predictions of the needle position between two images acquisitions.

\section{CONCLUSIONS AND FUTURE WORK}

In this paper we proposed a method to accurately predict the trajectory of a needle during insertion under lateral motion of the tissue. We proposed a 3D model of the flexible needle that allows to take into account the effect of the motion of the tissues on the needle shape. The model can give an accurate short term prediction of the needle motion. We demonstrated the advantage of updating the tissue model using visual feedback to reduce the prediction error of the model. The proposed algorithm based on the unscented
Kalman filter could give a good tracking of the tissue motion. Even if the algorithm requires the visual tracking of the 3D shape of the needle, we showed that the prediction error can be reduced even when using slow image acquisition systems.

Future work will address the test of the method using 3D ultrasound as visual feedback. The method should allow the reduction of the complexity of the tracking algorithm by providing an estimation of the needle position in the volume. The time update of the tissue motion used in the UKF will also be improved by using a more accurate model that can take into account the typical characteristics of real physiological motions. This should allow the reduction of the modeling error when the tissues are moving between two image acquisitions. We also plan to use the prediction provided by the model to design a predictive controller and use it in an image-guided closed-loop scheme to allow better targeting capabilities in moving tissues.

\section{REFERENCES}

[1] K. Reed, A. Majewicz, V. Kallem, R. Alterovitz, K. Goldberg, N. Cowan, and A. Okamura, "Robot-assisted needle steering," Robotics Automation Magazine, IEEE, vol. 18, pp. 35-46, Dec 2011.

[2] N. Abolhassani, R. Patel, and M. Moallem, "Needle insertion into soft tissue: A survey," Medical Engineering \& Physics, vol. 29, no. 4, pp. $413-431,2007$.

[3] R. Webster III, J. Kim, N. Cowan, G. Chirikjian, and A. Okamura, "Nonholonomic modeling of needle steering," The International Journal of Robotics Research, vol. 25, no. 5-6, pp. 509-525, 2006.

[4] S. Yamaguchi, K. Tsutsui, K. Satake, S. Morikawa, Y. Shirai, and H. Tanaka, "Dynamic analysis of a needle insertion for soft materials: Arbitrary lagrangianeulerian-based three-dimensional finite element analysis," Computers in Biology and Medicine, vol. 53, no. 0, pp. 42 $-47,2014$.

[5] M. Abayazid, R. Roesthuis, R. Reilink, and S. Misra, "Integrating deflection models and image feedback for real-time flexible needle steering," IEEE Trans. on Robotics, vol. 29, pp. 542-553, April 2013.

[6] B. Fallahi, M. Khadem, C. Rossa, R. Sloboda, N. Usmani, and M. Tavakoli, "Extended bicycle model for needle steering in soft tissue," in IEEE/RSJ Int. Conf. on Intelligent Robots and Systems, pp. 4375-4380, Sept 2015.

[7] S. Misra, K. Reed, B. Schafer, K. Ramesh, and A. Okamura, "Mechanics of flexible needles robotically steered through soft tissue," The International Journal of Robotics Research, 2010.

[8] Y. Zhou, K. Thiruvalluvan, L. Krzeminski, W. Moore, Z. Xu, and Z. Liang, "Ct-guided robotic needle biopsy of lung nodules with respiratory motion experimental system and preliminary test," The International Journal of Medical Robotics and Computer Assisted Surgery, vol. 9, no. 3, pp. 317-330, 2013.

[9] A. Majewicz, J. Siegel, A. Stanley, and A. Okamura, "Design and evaluation of duty-cycling steering algorithms for robotically-driven steerable needles," in IEEE Int. Conf. on Robotics and Automation, pp. 5883-5888, May 2014.

[10] P. Moreira, M. Abayazid, and S. Misra, "Towards physiological motion compensation for flexible needle interventions," in IEEE/RSJ Int. Conf. on Intelligent Robots and Systems, pp. 831-836, Sept 2015.

[11] S. Misra, K. Reed, B. W. Schafer, K. Ramesh, and A. Okamura, "Observations and models for needle-tissue interactions," in IEEE Int. Conf. on Robotics and Automation, pp. 2687-2692, May 2009.

[12] K. Reed, A. Okamura, and N. Cowan, "Modeling and control of needles with torsional friction," IEEE Trans. on Biomedical Engineering, vol. 56, pp. 2905-2916, Dec 2009.

[13] S. Julier and J. Uhlmann, "A new extension of the kalman filter to nonlinear systems," in AeroSense, vol. 3068, pp. 182-193, 1997.

[14] E. Wan and R. Van Der Merwe, "The unscented kalman filter for nonlinear estimation," in IEEE Adaptive Systems for Signal Processing, Communications, and Control Symposium, pp. 153-158, 2000.

[15] J. Chevrie, A. Krupa, and M. Babel, "Needle steering fusing direct base manipulation and tip-based control," in IEEE Int. Conf. on Robotics and Automation, pp. 4450-4455, May 2016.

[16] X. Pan, J. Gao, S. Tao, K. Liu, J. Bai, and J. Luo, "A two-step optical flow method for strain estimation in elastography: Simulation and phantom study," Ultrasonics, vol. 54, no. 4, pp. 990 - 996, 2014. 